\title{
COUNTING CYCLES IN LABELED GRAPHS: THE NONPOSITIVE IMMERSION PROPERTY FOR ONE-RELATOR GROUPS
}

\author{
JOSEPH HELFER AND DANIEL T. WISE
}

\begin{abstract}
We prove a rank 1 version of the Hanna Neumann Theorem. This shows that every one-relator 2-complex without torsion has the nonpositive immersion property. The proof generalizes to staggered and reducible 2-complexes.
\end{abstract}

\section{INTRODUCTION}

A deterministically labeled digraph $\Gamma$ is a nonempty graph whose edges are directed and labeled with the letters from an alphabet $\left\{a_{1}, a_{2}, \ldots\right\}$, with the additional property that at each vertex of $\Gamma$, no two outgoing edges have the same label, and no two incoming edges have the same label. These are termed inverse automata in the computer science literature. Let $w$ be a nonempty word in $\left\{a_{1}^{ \pm 1}, a_{2}^{ \pm 1}, \ldots\right\}$ that is reduced in the sense that no two consecutive letters of $w$ are inverse to each other, and the first and last letters of $w$ are not inverse to each other. We assume that $w$ is simple in the sense that $w \neq v^{p}$ for any word $v$ and $p>1$. A $w$-cycle in $\Gamma$ is a closed based path in $\Gamma$ whose label is of the form $w^{n}$ for some $n \geq 1$. Two $w$-cycles in $\Gamma$ are equivalent if there is a path with label $w^{m}$ joining their initial vertices for some $m \geq 1$. The number of equivalence classes of $w$-cycles in $\Gamma$ is denoted by $\overline{\#}_{w}(\Gamma)$. Finally, let $\beta_{1}(\Gamma)=\operatorname{rank}\left(\mathrm{H}_{1}(\Gamma)\right)$ be the first Betti number of $\Gamma$, and recall that $\beta_{1}(\Gamma)=|\mathcal{E}(\Gamma)|-|\mathcal{V}(\Gamma)|+1$ when $\Gamma$ is finite, connected, and nonempty. We use the notation $\mathcal{E}(Y)=\operatorname{Edges}(Y)$ and $\mathcal{V}(Y)=\operatorname{Vertices}(Y)$ for a complex $Y$.

In this paper we prove the following naive statement illustrated in Figure 1:

Theorem 1.1. Let $\Gamma$ be a deterministically labeled finite digraph, and let $w$ be a reduced simple word in its alphabet. Then $\overline{\#}_{w}(\Gamma) \leq \beta_{1}(\Gamma)$.

A proof is given at the end of Section 4.

Theorem 1.1 was conjectured in [Wis03, as part of a program to prove that every one-relator group is coherent. See Remark 4.5. Theorem 1.1 was proven when $w$ is a positive word in the sense that it has no $a_{i}^{-1}$ in [Wisb]. The inequality $\overline{\#}_{w}(\Gamma) \leq 2 \beta_{1}(\Gamma)$ was proven in Wis06] under the assumption that the Strengthened Hanna Neumann Conjecture holds. This latter conjecture was recently proven in [Fri15, Min12, Dic11]. The spirit of Dicks' proof which was extracted from Mineyev's argument, and the realization that orderability should play a critical role here, has

Date: September 27, 2018.

2010 Mathematics Subject Classification. 20F67, 20F65, 20 E06.

Key words and phrases. One-relator groups, orderable groups, inverse automata.

Research supported by NSERC. 


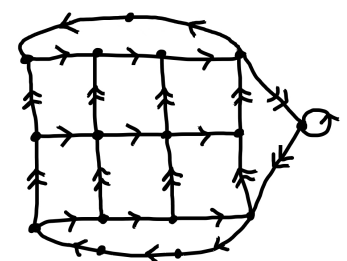

Figure 1. Some values of $\#_{w}$ and $\mathbb{\#}_{w}$ for a deterministically labeled digraph.

\begin{tabular}{c||c|c|c|c} 
& $w=a b a^{-1} b^{-1}$ & $w=b$ & $w=a^{30} b^{2} a^{30} b^{-2}$ & $w=a$ \\
\hline \hline$\#_{w}$ & 6 & 4 & 5 & 12 \\
$\overline{\#}_{w}$ & 6 & 1 & 5 & 3
\end{tabular}

inspired this note. The connection with the Strengthened Hanna Neumann Theorem and a sense in which this is a rank-one version of it is explained in Section 8, which can be read independently of the other sections.

Lars Louder and Henry Wilton have independently proven Theorem 1.1] in [LW14]. Their lovely proof is more geometrically palpable than ours, yet also relies on orderability in a fundamental way.

Definition 1.2. A 2-complex $X$ has nonpositive immersions if for every combinatorial immersion $Y \rightarrow X$ with $Y$ compact and connected, either $\chi(Y) \leq 0$ or $Y$ is contractible. We refer to Wisa] for a variety of classes of 2-complexes with nonpositive immersions.

The motivation for Theorem 1.1 is the following consequence which is a special case of Theorem 6.1:

Theorem 1.3. Let $X$ be a 2-complex with a single 2-cell whose attaching map is not homotopic to a path of the form $v^{n}$ where $n>1$ and $v \rightarrow X^{1}$ is a closed path. Then $X$ has nonpositive immersions.

Theorem 1.1 is a simplified statement of results simultaneously counting multiple types of immersed cycles in Theorems 4.1 and 5.5 . These apply to the class of "bislim" 2-complexes which include staggered 2-complexes, and to the more general class of "slim" 2-complexes (see Definition 2.1). In each case, an additional collapsing conclusion is obtained, showing that these 2-complexes have nonpositive immersions. In Section 6 we describe a way to treat the nonpositive immersion property for 2 complexes whose $\pi_{1}$ is not torsion-free. In Section 7 we show that adding a 1-cell and 2 -cell to a slim 2-complex usually results in another slim 2-complex. We deduce that Howie's reducible 2-complexes are slim when they have torsion-free $\pi_{1}$. Section 8 contains an algebraic reformulation and consequence of Theorem 1.1 .

\section{Definitions}

2.1. Preorder. A preorder on a set $E$ is a reflexive, transitive relation on $E$, denoted by $\preceq$. As usual, $a \prec b$ means that $(a \preceq b) \wedge \neg(b \preceq a)$. An element $a \in S$ is minimal in a subset $S \subseteq E$ if there is no $s \in S$ such that $s \prec a$. The element $a$ is strictly maximal in $S$ if there is no $s \in S-\{a\}$ such that $a \preceq s$. The reader should keep in mind the special case of a total ordering. 


\subsection{Slim and bi-slim 2-complexes.}

Definition 2.1 (Slim and bi-slim). A combinatorial 2-complex $X$ is slim if:

(1) There is a $\pi_{1} X$-invariant preorder on $\mathcal{E}(\widetilde{X})$.

(2) $\mathcal{E}(\partial \widetilde{R})$ has a unique strictly maximal edge $e_{\widetilde{R}}^{+}$for each 2-cell $\widetilde{R}$ of $\widetilde{X}$.

Moreover, $e_{\widetilde{R}}^{+}$is traversed exactly once by the boundary path $\partial_{\mathrm{p}} R$.

(3) If $\widetilde{R}_{1}$ and $\widetilde{R}_{2}$ are distinct 2-cells in $\widetilde{X}$ and $e_{\widetilde{R}_{1}}^{+}$lies in $\partial \widetilde{R}_{2}$ then $e_{\widetilde{R}_{1}}^{+} \prec e_{\widetilde{R}_{2}}^{+}$.

$X$ is bi-slim if it additionally satisfies:

(4) for each 2-cell $\widetilde{R}$ in $\widetilde{X}$, there is a distinguished edge $e_{\widetilde{R}}^{-}$such that for distinct 2-cells $\widetilde{R}_{1}$ and $\widetilde{R}_{2}$, if $\partial_{\mathrm{p}} \widetilde{R}_{1}$ traverses $e_{\widetilde{R}_{2}}^{-}$, then $e_{\widetilde{R}_{1}}^{+} \prec e_{\widetilde{R}_{2}}^{+}$.

Example 2.2. Let $\varphi: J \rightarrow J$ be a $\pi_{1}$-injective map from a graph to itself. We show that the mapping torus $X$ of $\varphi$ is slim. The attaching map of each 2-cell in $X$ is of the form $t_{u} a^{-1} t_{v}^{-1} \phi(a)$, where $a$ is a "vertical" edge arising from $J$ and each $t_{p}$ is a "horizontal" edge arising from a vertex $p$ of $J$. Let $\rho: \widetilde{X} \rightarrow \mathbb{R}$ be the map associated to the homomorphism $\pi_{1} X \rightarrow \mathbb{Z}$ induced by $\pi_{1} J \mapsto 0$ and $t_{p} \mapsto 1$. For edges $a, b$ of $\widetilde{X}$, we declare $a \preceq b$ if $\rho\left(a^{\prime}\right) \leq \rho\left(b^{\prime}\right)$, where $a^{\prime}, b^{\prime}$ are barycenters of $a, b$.

Definition 2.3. A 2-complex $X$ is staggered if its 2-cells are totally ordered and a subset of its edges are totally ordered in such a way that

(1) $\partial_{\mathrm{p}} R$ is immersed and traverses at least one ordered edge for each 2-cell $R$.

(2) If $R_{1}<R_{2}$ then $\min \left(R_{1}\right)<\min \left(R_{2}\right)$ and $\max \left(R_{1}\right)<\max \left(R_{2}\right)$, where $\min (R)$ and $\max (R)$ denote the minimal and maximal edges in $\partial R$.

In particular, the standard 2-complex $X$ of the presentation $\langle a, b, \ldots \mid W\rangle$ of a one-relator group without torsion is staggered and hence bi-slim by the following:

Proposition 2.4. Suppose $X$ is staggered and simple in the sense that no 2-cell is attached along a proper power. Then $X$ is bi-slim.

Proof. Since $\pi_{1} X$ is locally indicable [How82, it has a left-ordering $<$ by [BH72].

For each ordered 1-cell $c$ of $X$, a $\pi_{1} X$-invariant ordering of the cells of $\widetilde{X}$ mapping to $c$ is induced by choosing a distinguished lift $\tilde{c}$, and declaring $g_{1} \tilde{c} \prec g_{2} \tilde{c}$ when $g_{1}<g_{2}$. If $g_{1} \widetilde{c}$ and $g_{2} \widetilde{d}$ are ordered cells that are not in the same orbit, then we declare $g_{1} \widetilde{c} \prec g_{2} \widetilde{d}$ if their projections satisfy $c<d$. We have thus produced a $\pi_{1} X$ invariant total ordering on the edges of $\widetilde{X}$ that project to ordered edges of $X$, and so Property (1) holds, and $e_{\widetilde{R}}^{+}$exists for each $\widetilde{R}$. To see that Property (2) holds, observe that $\partial_{\mathrm{p}} \widetilde{R}$ embeds in $\widetilde{X}$ for each 2-cell $\widetilde{R}$. This is a well-known generalization of Weinbaum's Subword Theorem Wei72] (c.f. Corollary 7.7). Property (3) obviously holds when $\widetilde{R}_{1}, \widetilde{R}_{2}$ project to different 2-cells in $X$ since then $\max \left(R_{1}\right)<\max \left(R_{2}\right)$. Property (3) holds when $\widetilde{R}_{2}=g \widetilde{R}_{1}$ with $g \neq 1$, since $e_{\widetilde{R}_{2}}^{+}=g e_{\widetilde{R}_{1}}^{+} \neq e_{\widetilde{R}_{2}}^{+}$and so $e_{\widetilde{R}_{1}}^{+} \prec e_{\widetilde{R}_{2}}^{+}$by definition of $e_{\widetilde{R}_{2}}^{+}$.

Let $R$ denote a 2-cell of $X$ with $c=\max (R)$. Let $\tilde{c}$ denote the distinguished lift of $c$ declared above. Let $\widetilde{R}$ be the lift of $R$ satisfying $e_{\widetilde{R}}^{+}=\tilde{c}$. Let $\tilde{b}$ denote the distinguished lift of $b=\min (R)$. Let $\left\{h_{k} \tilde{b}\right\}$ be the translates of $\tilde{b}$ to edges of $\partial \widetilde{R}$. 
Consider the complete digraph $K$ with vertices $\left\{h_{k} \tilde{b}\right\}$ and with an edge directed from $h_{i} \tilde{b}$ to $h_{j} \tilde{b}$ if $h_{j}^{-1}<h_{i}^{-1}$. The ordering of $\pi_{1} X$ ensures that $K$ is acyclic. We declare $e_{\widetilde{R}}^{-}$to be the source $h_{s} \tilde{b}$ of $K$. For $g \in \pi_{1} X$ we declare $e_{g \widetilde{R}}^{-}=g e_{\widetilde{R}}^{-}$.

We now verify that Property (4) holds. If $\widetilde{R}_{1}, \widetilde{R}_{2}$ project to different cells in $X$, then $\min \left(R_{1}\right)<\min \left(R_{2}\right)$ and so $R_{1}<R_{2}$ and so $\max \left(R_{1}\right)<\max \left(R_{2}\right)$ and hence $e_{\widetilde{R}_{1}}^{+}<e_{\widetilde{R}_{2}}^{+}$. If $\widetilde{R}_{1}, \widetilde{R}_{2}$ project to the same cell $R$, then $e_{\widetilde{R}_{1}}^{+}=g_{1} \tilde{c}$ and $e_{\widetilde{R}_{2}}^{+}=g_{2} \tilde{c}$ for some $g_{1}, g_{2} \in \pi_{1} X$ where $\tilde{c}$ is the distinguished lift of $c=\max (R)$. Note that $e_{\widetilde{R}_{2}}^{-}=g_{2} e_{\widetilde{R}}^{-}=g_{2} h_{s} \tilde{b}$. Since $e_{\widetilde{R}_{2}}^{-}$lies in $\partial \widetilde{R}_{1}$ we also have $e_{\widetilde{R}_{2}}^{-}=g_{1} h_{j} \tilde{b}$ with $h_{j}^{-1}<h_{s}^{-1}$. Comparing coefficients we have $\left(g_{1} h_{j}\right)=\left(g_{2} h_{s}\right)$. We conclude that $e_{\widetilde{R}_{1}}^{+}<e_{\widetilde{R}_{2}}^{+}$since $g_{1}=\left(g_{1} h_{j}\right) h_{j}^{-1}<\left(g_{2} h_{s}\right) h_{s}^{-1}=g_{2}$.

The referee observed that the ordering arising from the complete digraph $K$ corresponds to the Duncan-Howie ordering for a one-relator group [DH91].

\section{3. $w$-cycles.}

Definition 2.5 (w-cycle). Let $X$ be a 2 -complex and $\left\{R_{1}, R_{2}, \ldots\right\}$ be its 2 -cells. For each $i$, let $w_{i} \rightarrow X^{1}$ be the immersed combinatorial circle corresponding to $\partial_{\mathrm{p}} R_{i}$. Let $\Gamma \rightarrow X^{1}$ be an immersion of a connected nonempty graph. A $w_{i}$-cycle in $\Gamma$ is a lift of $\widetilde{w}_{i}$ to $\Gamma$. Two such lifts are equivalent if they differ by an element of $\operatorname{Aut}\left(\widetilde{w}_{i}\right)$.

$\overline{\#}_{w_{i}}(\Gamma)$ is the number of equivalence classes of $w_{i}$-cycles in $\Gamma$ and $\#_{w}(\Gamma)=$ $\sum_{i} \#_{w_{i}}(\Gamma)$. Likewise, $\# w_{i}(\Gamma)$ is the number of $w_{i}$-cycles and $\#{ }_{w}(\Gamma)=\sum_{i} \#_{w_{i}}(\Gamma)$. Note that the use of the symbols $\#_{w}$ and $\overline{\#}_{w}$ is interchanged with respect to their use in [Wis06].

2.4. Pre-widges. We henceforth assume that $X$ is slim. The preorder on the $\mathcal{E}(\widetilde{X})$ induces a $\pi_{1} X^{1}$-invariant preorder on the $\mathcal{E}\left(\widetilde{X^{1}}\right)$ via the map $\widetilde{X^{1}} \rightarrow \widetilde{X}^{1}$.

Fixing basepoints of $X^{1}$ and $\Gamma$, we regard the universal cover $\widetilde{\Gamma}$ as a subtree of $\widetilde{X^{1}}$, and we restrict the above preorder to a $\pi_{1} \Gamma$-invariant preorder on the $\mathcal{E}(\widetilde{\Gamma})$.

A $w_{i}$-line is the image of a lift $\widetilde{w}_{i} \hookrightarrow \widetilde{\Gamma}$ of a $w_{i}$-cycle in $\Gamma$. We use the term $w$-line to indicate a $w_{i}$-line for some $i$.

Let $\ell$ be a $w$-line. A pre-widge of $\ell$ is an edge that is strictly maximal in $\mathcal{E}(\ell)$. A pre-widge is an edge of $\widetilde{\Gamma}$ which is a pre-widge of some $w$-line.

As $\pi_{1} \Gamma$ permutes the $w_{i}$-lines (for each $i$ ) and preserves the ordering, we see that $\pi_{1} \Gamma$ permutes the pre-widges. For each $w$-line $\ell$, the pre-widges of $\ell$ lie in a single $\mathrm{Stab}_{\pi_{1} X^{1}}(\ell)$-orbit by Definition 2.1.(2). No edge is a pre-widge of two different $w$-lines by Definition 2.1.(3).

\section{WIDGES AND ISLES}

The image in $\Gamma$ of a pre-widge is a widge. Let $\mathcal{W}(\Gamma) \subset \mathcal{E}(\Gamma)$ denote the set of widges in $\Gamma$. Removing the open edges $\mathcal{W}(\Gamma)$ from $\Gamma$, we obtain a set $\mathcal{I}(\Gamma)$ of components called isles. The significance of the isle-widge decomposition lies in:

Lemma 3.1. Let $X$ be slim. Let $\Gamma \rightarrow X^{1}$ be an immersion of a finite nonempty graph. Then $\#_{w}(\Gamma)<\beta_{1}(\Gamma)+T$, where $T$ is the number of isles that are trees. 
Proof. We first express the Euler characteristic of $\Gamma$ in terms of the decomposition:

$$
\chi(\Gamma)=\sum_{I \in \mathcal{I}(\Gamma)} \chi(I)-|\mathcal{W}(\Gamma)|
$$

hence

$$
|\mathcal{W}(\Gamma)|=-\chi(\Gamma)+\sum_{I \in \mathcal{I}(\Gamma)} \chi(I)=\beta_{1}(\Gamma)-1+\sum_{I \in \mathcal{I}(\Gamma)} \chi(I)
$$

Since the last term is less than or equal to $T$, we have $|\mathcal{W}(\Gamma)|<\beta_{1}(\Gamma)+T$. Finally, $\#_{w}(\Gamma)=|\mathcal{W}(\Gamma)|$ by the definition of widge.

\section{Counting $w$-CyCles With multiplicity in the Bi-Slim CASE}

Theorem 4.1. Let $X$ be bi-slim. Let $\Gamma \rightarrow X^{1}$ be an immersion of a finite nonempty graph. Suppose each edge of $\Gamma$ is traversed by at least two $w$-cycles or traversed at least twice by some $w$-cycle. Then either $\Gamma$ is a single vertex or

$$
\#{ }_{w}(\Gamma)<\beta_{1}(\Gamma) .
$$

Proof. By Lemma 3.1, the desired conclusion will follow by showing that no isle $I$ of $\Gamma$ is a tree. Suppose $I$ were a tree, and consider $\widetilde{I} \subset \widetilde{X^{1}}$. Choose $e$ to be a pre-widge of a $w$-line $\ell$ intersecting $\widetilde{I}$, and assume that $e$ is minimal among all such choices. Observe that the edges $e_{1}, e_{2}$ in $\ell$ immediately before and after $\ell \cap \widetilde{I}$ are pre-widges of $\ell$. Indeed, each $e_{i}$ is a pre-widge since it is incident to $\widetilde{I}$, and if $e_{i}$ were a pre-widge of another line, then $e_{i} \prec e$ by Definition 2.1.(3), violating the minimality of $e$. Observe that neither $e_{i}$ maps to the distinguished edge $e_{\widetilde{R}}^{\bar{R}}$ of the 2-cell in $\widetilde{X}$ to whose boundary $\ell$ maps. Indeed, a second $w$-line $\ell^{\prime}$ traversing $e_{i}$ would have a widge $q$ satisfying $q \prec e$ by Definition 2.1.(4), contradicting the minimality of $\ell$. Thus the arc connecting $e_{1}, e_{2}$ contains an edge $e^{-}$mapping to $e_{\widetilde{R}}^{-}$. Let $\ell^{\prime}$ be another $w$-line that traverses $e^{-}$. Then $\ell^{\prime}$ intersects $\widetilde{I}$ and $e^{\prime} \prec e$ for any pre-widge $e^{\prime}$ of $\ell^{\prime}$ by Definition 2.1.(4). This contradicts the minimality of $e$.

Definition 4.2 (Collapsing $\Gamma^{w}$ ). We form a 2 -complex $\Gamma^{w}$ from $\Gamma$ by adding a single 2-cell for a representative of each $w$-cycle equivalence class in $\Gamma$.

An edge $e$ in a 2-complex is a free face of a 2-cell $f$ if $\partial_{\mathrm{p}} f$ traverses $e$ exactly once and $e$ is not in the boundary of any other 2-cell. In this case, we can collapse to a subcomplex with the same homotopy type by removing the open cells $e, f$. If $B$ is obtained from $A$ by a sequence of such collapses then we say that $A$ collapses to $B$.

Corollary 4.3. Let $X$ be bi-slim. Let $\Gamma \rightarrow X^{1}$ be an immersion of a finite nonempty graph. Then $\overline{\#}_{w}(\Gamma) \leq \beta_{1}(\Gamma)$ with equality only if $\Gamma^{w}$ collapses to a tree.

Proof. Suppose $\Gamma^{w}$ has an edge $e$ that is isolated in the sense that $e$ is not in the boundary of any 2-cell. The statement holds for (each component of) $\Gamma-e$ by induction on the number of such edges. When $e$ is non-separating, the extra $\beta_{1}$ yields a strict inequality for $\Gamma$. When $e$ is separating, either both components of $\Gamma^{w}-e$ collapse to a tree, or we get a strict inequality for one of the components of $\Gamma-e$ and hence for $\Gamma$.

We now assume that $\Gamma^{w}$ has no isolated edge. Suppose $\Gamma^{w}$ has a free face $e$. Observe that $e$ cannot be separating. Removing $e$ decreases both $\overline{\#}_{w}$ and $\beta_{1}$ by 1 . 
Hence, the result holds for $\Gamma$ by induction on the number of edges. The base-case of this induction holds by Theorem 4.1 , since $\#_{w}(\Gamma) \leq \#_{w}(\Gamma)$.

Proof of Theorem 1.1. A deterministically labeled digraph $\Gamma$, as described in the introduction, is equivalent to a combinatorial immersion of a graph into a bouquet of circles $X^{1}$. Attaching a 2-cell to $X^{1}$ along a path corresponding to the cyclically reduced simple word $w$ produces a bi-slim complex by Proposition 2.4. There is a bijection between $w$-cycles in the sense of the introduction and $w$-cycles in the sense of Definition 2.5, and this bijection respects the equivalence relations. Theorem 1.1 then follows immediately from Corollary 4.3. Moreover, under the additional assumption that each edge of $\Gamma$ lies in at least two $w$-cycles, Theorem 4.1 gives the stronger inequality $\#{ }_{w}(\Gamma)<\beta_{1}(\Gamma)$.

Corollary 4.4. If $X$ is bi-slim, then $X$ has nonpositive immersions.

Proof. Let $Y \rightarrow X$ be an immersion with $\chi(Y)>0$. Let $\Gamma=Y^{1}$. Let $F$ be the number of 2-cells in $Y$. The first inequality in 20 holds by Corollary 4.3 , the second holds since $\chi(Y)=1-\beta_{1}(\Gamma)+F$, and the third holds by definition of $F$ and $\#_{w}$.

$$
\#{ }_{w}(\Gamma) \leq \beta_{1}(\Gamma) \leq F \leq \#{ }_{w}(\Gamma)
$$

Thus each inequality in (2) is an equality, and so $Y=\Gamma^{w}$ collapses by the second statement of Corollary 4.3 .

Remark 4.5 (Coherence of one-relator groups). The original motivation for counting $w$-cycles, and in particular for proving Corollary 4.4, is to affirmatively answer G. Baumslag's question on the coherence of one-relator groups. There is currently a gap in the proof of the main goal of Wisa which asserts: if $X$ has nonpositive immersions then $\pi_{1} X$ is coherent in the sense that every finitely generated subgroup of $\pi_{1} X$ is finitely presented.

\section{Counting $w$-CyCles (Without multiplicity) in the Slim Case}

As we now only count equivalence classes of $w$-cycles, we focus on only one widge from each equivalence class of $w$-cycles. Accordingly, two widges in $\Gamma$ are equivalent if they are images of pre-widges of a common $w$-line. We arbitrarily select one widge from each equivalence class and call these great widges and refer to their preimages as great pre-widges. The great isles are the components obtained by removing the great widges from $\Gamma$.

Lemma 5.1. Let $X$ be slim. Let $\Gamma \rightarrow X^{1}$ be an immersion of a finite nonempty graph. Then $\overline{\#}_{w}(\Gamma)<\beta_{1}(\Gamma)+\bar{T}$, where $\bar{T}$ is the number of great isles that are trees.

Proof. This is proved like Lemma 3.1 replacing $\#{ }_{w}(\Gamma)$ by $\overline{\#}_{w}(\Gamma)$ and $T$ by $\bar{T}$.

A great widge is a local widge to a great isle if its corresponding $w$-cycle does not traverse an edge in any other great isle.

Lemma 5.2. Let $\Gamma \rightarrow X$ be an immersion of a locally finite, connected graph. Let $I$ be a great isle that is a finite tree. Let $J$ be the union of $I$ and its local widges. If $\pi_{1} J \rightarrow \pi_{1} X$ has trivial image, then $I$ is the unique great isle. 
Proof. Consider $\widetilde{J} \subset \widetilde{\Gamma}$. Since $\operatorname{Stab}(\widetilde{J})$ is a subgroup of $\operatorname{ker}\left(\pi_{1} X^{1} \rightarrow \pi_{1} X\right)$ we see that there are finitely many $\preceq$-equivalence classes of edges incident with $\widetilde{J}$ in $\widetilde{\Gamma}$.

Let $\tilde{e}_{1}$ be a minimal great pre-widge with a single vertex in $\widetilde{J}$; its image $e_{1}$ is not a local widge. Consider its $w$-line $\ell_{1}$. Since $e_{1}$ is not a local widge of $I$, the line $\ell_{1}$ contains another great pre-widge $\tilde{e}_{2}$ with a single vertex in $\widetilde{J}$. Since $\tilde{e}_{2}$ is on $\ell_{1}$, we must have $\tilde{e}_{2} \preceq \tilde{e}_{1}$. The minimality of $\tilde{e}_{1}$ obviates $\tilde{e}_{2} \prec \tilde{e}_{1}$.

If $\tilde{e}_{1} \preceq \tilde{e}_{2}$ then $\tilde{e}_{2}$ is a pre-widge of $\ell_{1}$ since $\tilde{e}_{1}$ is a pre-widge. As $\tilde{e}_{2}$ is a great pre-widge, it must be a great pre-widge of $\ell_{1}$ and hence in the same $\operatorname{Stab}\left(\ell_{1}\right)$-orbit as $\tilde{e}_{1}$. But then $\tilde{e}_{1}$ and $\tilde{e}_{2}$ have the same image in $\Gamma$, which is thus a local widge as the path from $\tilde{e}_{1}$ to $\tilde{e}_{2}$ is in $\widetilde{J}$. This contradicts that $\tilde{e}_{1}$ is not a local widge.

As each great widge incident to $I$ is local, we see that $I$ is the only isle.

Lemma 5.3. Let $\Gamma \rightarrow X$ be an immersion of a locally finite, connected graph. If some great isle is a finite tree, then it is the only great isle.

Proof. Let $\Gamma$ be a counterexample that is minimal in the sense that it has a tree great isle $I$ with fewest local widges among all tree great isles of all counterexamples.

Let $J$ be the union of $I$ with all its local widges. Observe that $\pi_{1} J \rightarrow \pi_{1} X$ has nontrivial image, for otherwise, Lemma 5.2 implies that $I$ is the only great isle.

Let $\widehat{\Gamma} \rightarrow \Gamma$ be the cover corresponding to $\operatorname{ker}\left(\pi_{1} \Gamma \rightarrow \pi_{1} X\right)$. Each $w$-cycle of $\Gamma$ lifts to a $w$-cycle of $\widehat{\Gamma}$, and moreover, every $w$-cycle of $\widehat{\Gamma}$ arises in this way. We can thus choose the great widges of $\widehat{\Gamma}$ to be the pre-images of great widges of $\Gamma$. Hence any pre-image $\widehat{I}$ of $I$ is a great isle of $\widehat{\Gamma}$. Since $\widehat{I}$ has fewer local widges than $I$ we obtain a smaller counterexample $\widehat{\Gamma}$.

Lemma 5.4. Let $\Gamma \rightarrow X$ be an immersion of a finite connected graph. If $\Gamma$ has a great isle that is a finite tree, then $\Gamma^{w}$ collapses to a tree.

Proof. We first verify that $\pi_{1} \Gamma \rightarrow \pi_{1} X$ is trivial. Indeed, otherwise, the corresponding cover $\widehat{\Gamma}$ has $\operatorname{deg}(\widehat{\Gamma} \rightarrow \Gamma)$ finite tree great isles, violating Lemma 5.3 .

Since every widge in $\Gamma$ is local to its unique tree great isle, we conclude, as in the proof of Lemma 5.2 , that there are finitely many $\preceq$-equivalence classes of edges in $\widetilde{\Gamma}$. Let $\tilde{e}$ be a maximal pre-widge in $\widetilde{\Gamma}$. Then by maximality, $\tilde{e}$ cannot lie in another $w$-line. Hence its image $e$ in $\Gamma$ is a free face in $\Gamma^{w}$ and the 2-cell on which $e$ lies can be collapsed. Repeating this process, we collapse $\Gamma^{w}$ to a tree.

Theorem 5.5. Let $X$ be slim. Let $\Gamma \rightarrow X^{1}$ be an immersed finite connected nonempty graph. Then:

$$
\overline{\#}_{w}(\Gamma) \leq \beta_{1}(\Gamma)
$$

Moreover, if $\overline{\#}_{w}(\Gamma)=\beta_{1}(\Gamma)$, then $\Gamma^{w}$ collapses to a tree.

Proof. By Lemma 5.3 , at most one great isle of $\Gamma$ is a tree. Hence $\overline{\#}_{w}(\Gamma) \leq \beta_{1}(\Gamma)$ by Lemma 5.1. The "moreover" part follows from Lemma 5.4 .

Corollary 5.6. If $X$ is slim, then $X$ has nonpositive immersions.

Proof. Replace Corollary 4.3 by Theorem 5.5 in the proof of Corollary 4.4 


\section{NONPOSITIVE IMMERSIONS WHEN THERE IS TORSION}

There are various ways of obtaining a version of nonpositive immersions for a one-relator group with torsion. Since one-relator groups with torsion are virtually torsion free (this holds more generally for staggered presentations where all relators are proper powers) the following provides a useful interpretation:

Theorem 6.1. Let $X_{*}$ be a 2-complex whose $i$-th 2-cell has attaching map $w_{i}^{n_{i}}$ where $w_{i}$ is not a proper power. Let $X$ be the 2 -complex with $X^{1}=X_{*}^{1}$ and whose $i$-th 2-cell has attaching map $w_{i}$. Let $\widehat{X}_{*} \rightarrow X_{*}$ be a finite regular cover such that no 2 -cell is attached along a proper power. Let $Z$ be a subcomplex of $\widehat{X}_{*}$ that contains exactly one 2-cell from each set of $n_{i} 2$-cells attached along a lift of $w_{i}^{n_{i}}$.

If $X$ is slim then $Z$ has nonpositive immersions.

If $X$ is bi-slim, and let $n=\min _{i}\left(n_{i}\right)$. Then for any collapsed immersion $Y \rightarrow Z$, either $Y$ has an isolated edge, or $Y$ is a vertex, or $\chi(Y) \leq-(n-1)|2-c e l l s(Y)|$.

Proof. Let $Y \rightarrow Z$ be an immersion with $Y$ compact and connected. Then the induced map $Y^{1} \rightarrow X$ is also an immersion, and each $w$-cycle in $Y^{1}$ with respect to $Z$ is also a $w$-cycle with respect to $X$. If $X$ is slim, then Theorem 5.5 holds for $X$, and it follows that the conclusion of Theorem 5.5 holds for $Z$ as well, and hence that $Z$ has nonpositive immersions (by the proof of Corlloary 5.6).

In the bi-slim case we note that, not only are the $w$-cycles of $Y^{1}$ with respect to $Z$ also $w$-cycles with respect to $X$, but each $w_{i}$-cycle in $Y$ appears with multiplicity at least $n_{i}$. Since $X$ is bi-slim, if $Y$ is not a single vertex and has no isolated edge, then by Theorem 4.1 we have:

$$
n|2-\operatorname{cells}(Y)|<\beta_{1}\left(Y^{1}\right)
$$

and hence

$$
\chi(Y)=\left(1-\beta_{1}\left(Y^{1}\right)\right)+|2-\operatorname{cells}(Y)| \leq(-n+1) \mid 2 \text {-cells }(Y) \mid .
$$

\section{Reducible is SLim}

Definition 7.1 (Enlargement). The connected combinatorial 2-complex $Y$ is an $(R, e)$-enlargement of the subcomplex $X$ if $Y-X=R \cup e$ where $e$ is an open edge, and $R$ is an open 2-cell, and $\partial_{\mathrm{p}} R$ traverses $e$ but $\partial_{\mathrm{p}} R$ is not homotopic in $X \cup e$ to a path traversing $e$ fewer times. Similarly, $Y$ is an e-enlargement if $Y-X$ consists of a single open edge $e$. An enlargement is simple if either it is an $e$-enlargement, or it is an $(R, e)$-enlargement and $\partial_{\mathrm{p}} R$ is not homotopic to a proper power in $X \cup e$.

Howie provided the following generalization of Weinbaum's subword theorem [How82, Cor 3.4]:

Lemma 7.2. Let $Y$ be an $(R, e)$-enlargement of $X$. Suppose $\partial_{\mathrm{p}} R=P_{1} P_{2}$ where each $P_{i}$ is a closed path in $Y$ that traverses e. Then each $P_{i}$ is essential in $Y$.

Lemma 7.3. Let $Y$ be an $(R, e)$-enlargement of $X$. Suppose $\pi_{1} Y$ is left-orderable. Then $X \rightarrow Y$ is $\pi_{1}$-injective on each component of $X$.

Proof. Arguing by contradiction, consider a minimal area disk diagram $D \rightarrow Y$ where $\partial_{\mathrm{p}} D$ is an essential closed path in $X$. Choose a lift $D \rightarrow \widetilde{Y}$ to the universal 
cover of $Y$. We order the edges in $\tilde{Y}$ mapping to $e$ by setting $g_{1} \tilde{e}<g_{2} \tilde{e}$ if $g_{1}<g_{2}$. Let $f$ be a maximal edge in image $(D \rightarrow \widetilde{Y})$ among all edges mapping to $e$. Let $f^{\prime}$ be an edge of $D$ mapping to $f$. Observe that $f^{\prime} \subset$ interior $(D)$ since $f^{\prime} \not \subset \partial D$ as $e \not \subset X$. Let $S^{\prime}, T^{\prime}$ denote the two 2-cells of $D$ on opposite sides of $f^{\prime}$, and let $S, T$ denote their images in $\widetilde{Y}$. We will show below that $S \neq T$. Let $g \in \pi_{1} Y$ be the nontrivial element such that $g S=T$. Both $g f$ and $g^{-1} f$ lie in image $(D \rightarrow \widetilde{Y})$. This contradicts the maximality of $f$ since either $g f>f$ or $g^{-1} f>f$.

We now reach a contradiction if $S=T$. Consider the edges mapping to $f$ in a lift $\partial_{\mathrm{p}} R \rightarrow \widetilde{Y}$. If there is only one such edge, then $S^{\prime}, T^{\prime}$ form a cancelable pair, and so the minimality of $D$ is violated. If two of these edges are oriented in the same way around $\partial_{\mathrm{p}} R$, then the subpath joining their initial vertices violates Lemma 7.2. Thus exactly two edges of $\partial_{\mathrm{p}} R$ map to $f$ so $\partial_{\mathrm{p}} R \rightarrow \tilde{Y}$ is of the form $\sigma f \sigma^{\prime} f^{-1}$. Moreover, projecting to $Y$, we find that $\sigma$ cannot traverse $e$, for it would violate Lemma 7.2 and likewise for $\sigma^{\prime}$. We conclude that $\partial_{\mathrm{p}} R$ is of the form $\sigma e \sigma^{\prime} e^{-1}$ where $\sigma$ and $\sigma^{\prime}$ are closed essential paths in $X$. However, in this case, $\pi_{1} Y$ splits as an HNN extension or amalgamated product (along $\langle\sigma\rangle$ ), depending on whether or not $X$ is connected, and hence $X \rightarrow Y$ is $\pi_{1}$-injective on each component.

Theorem 7.4. Let $Y$ be a simple enlargement of $X$. Then $Y$ is slim if each component of $X$ is slim.

Proof. As each component of $X$ is slim, it has nonpositive immersions by Corollary 5.6. And hence has locally indicable $\pi_{1}$ by Wisa. As the enlargement is simple, $\pi_{1} Y$ is locally indicable [How82, Cor 4.2] and thus has a left-ordering $<$. For an $(R, e)$-enlargement, any proper nontrivial subpath of $\partial_{\mathrm{p}} R$ which starts and ends at an initial vertex of $e$ is essential in $\pi_{1} Y$ by Lemma 7.2 and our assumption that $\partial_{\mathrm{p}} R$ cannot be homotoped to traverse $e$ fewer times. Furthermore, as $X \rightarrow Y$ is $\pi_{1}$-injective on each component by Lemma 7.3, we see that any subpath of $\partial_{\mathrm{p}} R$ that starts and ends at an initial vertex of $e$ is essential in $\pi_{1} Y$.

To see that $Y$ is slim, we declare a $\pi_{1} Y$-invariant preorder on the edges of $\widetilde{Y}$ as follows: $\tilde{c}_{1} \preceq \tilde{c}_{2}$ if either:

(1) $\tilde{c}_{1}$ maps to $X$ and $\tilde{c}_{2}$ maps to $e$.

(2) $\tilde{c}_{1}=g \tilde{c}_{2}$ with $g<1_{\pi_{1} Y}$ and both $\tilde{c}_{1}$ and $\tilde{c}_{2}$ map to $e$.

(3) $\tilde{c}_{1}, \tilde{c}_{2}$ lie in the same component of the pre-image of $X$ and $\tilde{c}_{1} \preceq \tilde{c}_{2}$ with respect to the slim structure on $X$.

Definition 7.5 (Reducible). A 2-complex $X=\cup_{i=0}^{m} X_{i}$ is [simply] reducible if $X_{0}$ is a vertex, and $X_{i+1}$ is a [simple] enlargement of $X_{i}$ for each $i \geq 0$. We allow $m=\infty$.

Howie's original definition of reducible 2-complex is a bit more general, as he imposes the laxer requirement that for an $(R, e)$-enlargement, $\partial_{\mathrm{p}} R$ is not homotopic in $X \cup e$ to a path not traversing $e$. However, any 2-complex satisfying Howie's definition has the homotopy type of a reduced 2-complex in the above sense.

Corollary 7.6. Every simply reducible 2-complex is slim.

Proof. This follows by induction from Theorem 7.4. Note that the preorder on the edges in copies of $\widetilde{X}_{i}$ are in agreement with the preorder on $\widetilde{X}_{i+1}$ for each $i$, and hence the case $m=\infty$ holds as well. 


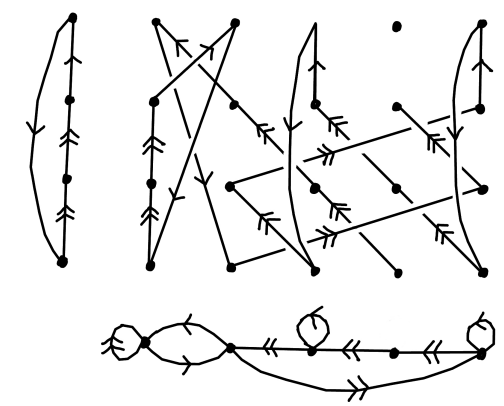

FiguRe 2. The above fiber product of a graph and a circle contains an arc, an isolated vertex, and two cycles with multiplicities one and two.

An $(R, e)$-enlargement $X_{i+1}$ of $X_{i}$ is tight if each subpath of $\partial_{\mathrm{p}} R$ mapping to $X_{i}$ lifts to an embedding in $\widetilde{X}_{i}$. And $X$ is a tight reducible complex if each $(R, e)$ enlargement $X_{i} \subset X_{i+1}$ is tight. The following is then a consequence of Lemma 7.3 .

Corollary 7.7. If $X$ is a tight reducible complex, then the boundary path of each 2-cell embeds in $\tilde{X}$.

\section{Connection to the Strengthened Hanna Neumann Theorem}

In this section, we describe the connection of Theorem 1.1 to the Strengthened Hanna Neumann Theorem.

Definition 8.1 (Fiber product). Let $\Gamma_{1}, \Gamma_{2}$ be labeled digraphs. Their fiber product is the labeled digraph whose vertices are pairs $\left(v_{1}, v_{2}\right)$ of vertices in $\Gamma_{1}, \Gamma_{2}$, and whose edges are pairs $\left(e_{1}, e_{2}\right)$ of edges in $\Gamma_{1}, \Gamma_{2}$ with the same label. The initial and terminal vertex of $\left(e_{1}, e_{2}\right)$ are $\left(u_{1}, u_{2}\right)$ and $\left(v_{1}, v_{2}\right)$ where $u_{i}, v_{i}$ are the initial and terminal vertices of $e_{i}$ in $\Gamma_{i}$.

Fiber products were popularized in combinatorial group theory by Stallings [Sta83]. The same construction, phrased in the language of finite state automata, was widely used in computer science to compute the intersection of regular languages.

Definition 8.2. The reduced rank of a graph $K$ is $\widetilde{\operatorname{rank}}(K)=\max \left\{\beta_{1}(K)-1,0\right\}$.

The Strengthened Hanna Neumann Theorem is equivalent to the following inequality, which was first stated explicitly by Walter Neumann in [Neu90. It is this statement that was proven in [Fri15, Min12].

Theorem 8.3. Let $\Gamma_{1}, \Gamma_{2}$ be connected deterministically labeled digraphs. Then:

$$
\sum_{K \in \text { Components }\left(\Gamma_{1} \otimes \Gamma_{2}\right)} \widetilde{\operatorname{rank}}(K) \leq \widetilde{\operatorname{rank}}\left(\Gamma_{1}\right) \cdot \widetilde{\operatorname{rank}}\left(\Gamma_{2}\right)
$$

Letting $\Gamma_{1}$ be the cycle labeled by a cyclically reduced word $w$ which is not a proper power, and letting $\Gamma_{2}$ be arbitrary, Theorem 1.1 can be restated as

$$
\sum_{K \in \text { Components }\left(\Gamma_{1} \otimes \Gamma_{2}\right)} \beta_{1}(K) \leq \beta_{1}\left(\Gamma_{1}\right) \cdot \beta_{1}\left(\Gamma_{2}\right)
$$


Unfortunately, Theorem 8.3 sheds no light on Theorem 1.1 since $\widetilde{\operatorname{rank}}\left(\Gamma_{1}\right)=0$ when $\Gamma_{1}$ is a cycle, and as illustrated in Figure 2, each component $K$ of $\Gamma_{1} \otimes \Gamma_{2}$ has $\widetilde{\operatorname{rank}}(K)=0$, so Equation (4) becomes $0 \leq 0$. Nevertheless, this connection was exploited in Wis06] to obtain a partial result by choosing $\Gamma_{1}$ appropriately related to $w$ so that no component $K$ has $\chi(K)=0$.

In parallel to the statement of the (Strengthened) Hanna Neumann Theorem which was originally formulated in terms of intersections of subgroups of a free group, we have the following:

Corollary 8.4 (Restatement of Theorem 1.1). Let $H$ be a finitely generated subgroup of a free group $F$. Let $Z \subset F$ be a maximal cyclic subgroup. Then the number of distinct conjugates of $Z$ that intersect $H$ nontrivially is bounded by $\operatorname{rank}(H)$.

Proof. Let $F=\pi_{1} B$ where $B$ is a bouquet of circles. Let $\widehat{B} \rightarrow B$ be the based covering space with $\pi_{1} \widehat{B}=H$. Let $\Gamma \subset \widehat{B}$ be a finite connected based subgraph. Direct and label the edges of $B$, and pull this back so $\Gamma$ is a finite deterministically labeled digraph. Let $Z=\langle w\rangle$, where we may assume without loss of generality that $w$ is cyclically reduced. Each conjugate of $Z$ that intersects $H$ nontrivially corresponds to a closed lift of some power $w^{n}$ of the path $w \rightarrow B$ at some vertex of $\Gamma$, and hence to a based $w$-cycle. Two based $w$-cycles in the same equivalence class correspond to vertices connected by a lift of a path $w^{k}$, and hence to the same conjugate of $Z$. The bound holds by Theorem 1.1 .

Corollary 8.5. Let $H$ be a finitely generated subgroup of a free group $F$. Suppose $H$ is isolated in the sense that $h^{p} \in F$ implies $h \in F$ for any $h \in F$ and $p>0$. Suppose $H g_{1}, \ldots, H g_{n}$ are distinct cosets with $n>\operatorname{rank}(H)$. Then:

$$
\bigcap_{i=1}^{n} g_{i}^{-1} H g_{i}=\left\{1_{F}\right\}
$$

Proof. Consider a nontrivial element $w \in \bigcap_{i=1}^{n} g_{i}^{-1} H g_{i}$. As $H$ is isolated, we may assume that $w$ is not a proper power. Let $Z=\langle w\rangle$, and apply Corollary 8.4 to $\left\{g_{i} Z g_{i}^{-1}: 1 \leq i \leq n\right\}$. Note that the maximal cyclic subgroup $Z$ is malnormal and so the conjugates are distinct.

Acknowledgment: We are grateful to the referees for many helpful corrections.

\section{REFERENCES}

[BH72] R. G. Burns and V. W. D. Hale. A note on group rings of certain torsion-free groups. Canad. Math. Bull., 15:441-445, 1972.

[DH91] Andrew J. Duncan and James Howie. The genus problem for one-relator products of locally indicable groups. Math. Z., 208(2):225-237, 1991.

[Dic11] Warren Dicks. Simplified Mineyev.http://mat.uab.es/ dicks/SimplifiedMineyev.pdf 2011.

[Fri15] Joel Friedman. Sheaves on graphs, their homological invariants, and a proof of the Hanna Neumann conjecture: with an appendix by Warren Dicks. Mem. Amer. Math. Soc., 233(1100):xii+106, 2015.

[How82] James Howie. On locally indicable groups. Math. Z., 180(4):445-461, 1982.

[LW14] Lars Louder and Henry Wilton. Stackings and the $W$-cycles conjecture. pages 1-9, 2014. 
[Min12] Igor Mineyev. Submultiplicativity and the Hanna Neumann conjecture. Ann. of Math. (2), 175(1):393-414, 2012.

[Neu90] Walter D. Neumann. On intersections of finitely generated subgroups of free groups. In Groups-Canberra 1989, pages 161-170. Springer, Berlin, 1990.

[Sta83] John R. Stallings. Topology of finite graphs. Invent. Math., 71(3):551-565, 1983.

[Wei72] C. M. Weinbaum. On relators and diagrams for groups with one defining relation. Illinois J. Math., 16:308-322, 1972.

[Wisa] Daniel T. Wise. Coherence, local-indicability, and nonpositive immersions. Available at http://www.math.mcgill.ca/wise/papers. Preprint.

[Wisb] Daniel T. Wise. Positive one-relator groups are coherent. Available at http://www.math. mcgill.ca/wise/papers pp. 1-19.

[Wis03] Daniel T. Wise. Nonpositive immersions, sectional curvature, and subgroup properties. Electron. Res. Announc. Amer. Math. Soc., 9:1-9 (electronic), 2003.

[Wis06] Daniel T. Wise. The coherence of one-relator groups with torsion and the Hanna Neumann conjecture. Bull. LMS, 2006.

Dept. of Math. \& Stats., McGill Univ., Montreal, QC, Canada H3A 0B9

E-mail address: joseph@helfer.ca wise@math.mcgill.ca 